\title{
Study on Electrical Properties of Vanadium Dioxide Thin Films Prepared by Sol-Gel Method
}

\author{
Ang $\mathrm{Li}^{1, \mathrm{a}}$, Qingguo Wang ${ }^{1, \mathrm{~b}}$, Wei Cheng ${ }^{1}$ and Yan Wang ${ }^{1}$ \\ ${ }^{1}$ Institute of Electrostatic and Electromagnetic Protection, \\ Ordnance Engineering College, Shijiazhuang 050003, China \\ a917370828@qq.com, 'pwang_99@hotmail.com
}

Keywords: electromagnetic protection, $\mathrm{VO}_{2}$ thin films, Sol-Gel, field-induced phase transition, conductivity.

\begin{abstract}
In order to develop environmental self-adaptation electromagnetic protection materials, $\mathrm{VO}_{2}$ thin films with different thickness have been prepared on high-purity single-crystal Si substrates by Sol-Gel method. A phase transition testing system was manufactured based on static high voltage source and copper electrode fixture. The field-induced phase transition of $\mathrm{VO}_{2}$ thin films has been researched through the variation of electric field intensity. The results show that the materials express an obvious MIT feature at $600 \mathrm{kV} / \mathrm{m}$. The variation of conductivity reaches more than 2.6 magnitudes at $700 \mathrm{kV} / \mathrm{m}$ and far from saturated. And there is still much room with the increase of external electric field.
\end{abstract}

\section{Introduction}

With the development of electronic information equipment, power system towards highly integrated, small size, low power consumption direction, advanced high-power radar and other frequency equipment widely deployed, especially with a growing potential threat in the high-power microwave (HPM ) [1], ultra wideband (UWB)[2] represented by the electromagnetic pulse weapons, leading to an increasingly complex battlefield electromagnetic environment. Electromagnetic protection [3] materials as the cornerstone of electromagnetic weaponry protection have been playing an irreplaceable role and become an important branch in the field of electromagnetic compatibility and protection. Conventional electromagnetic protection materials, mainly isolated electromagnetic interference and electronic equipment in the physical level, this passive protection method can not process the contradiction protection between the send and receive of information under normal environment and strong electromagnetic interference. F.J.Morin [4]first discovered the vanadium oxides [5] having a phase transition and the conductive switching characteristics in the United States Bell Labs at the year of 1959,of which the vanadium dioxide showing a reversible MIT at 68 degrees with a sudden change of optical and electrical constant has become a research focus. Thin film morphology of vanadium dioxide can overcome the disadvantages of its single crystal state can easily crack [6] after multiple cycles of phase transition. Vanadium dioxide thin films have wide application prospects among the fields of conductive materials, architectural smart windows [7,8], and laser protection [9] devices. Present research mainly focused on phase transition by thermal excitation trigger, however the conductive switching characteristics of vanadium dioxide films under strong electric field environment from the perspective of electromagnetic protection were rarely reported.

In this work, we prepared $\mathrm{VO}_{2}$ thin films with different thickness on high-purity single-crystal $\mathrm{Si}$ substrates via Sol-Gel followed by thermal annealing. The chemical composition and microcosmic morphology of the films were characterized by X-ray diffractometer and scanning electron microscopy. An electrical characteristics testing system based on static high voltage source and copper electrode fixture was manufactured in order to study the MIT properties of $\mathrm{VO}_{2}$ thin films under strong electric field. The experimental results provide a reference to the design and test of electromagnetic protection materials. 


\section{Experimental}

The aqueous sol was prepared using the method directed by document[10], heating $5 \mathrm{~g}$ of $\mathrm{V}_{2} \mathrm{O}_{5}$ power crucible into the temperature of $880^{\circ} \mathrm{C}$ and kept the temperature until the power completely molten then pouring it into $300 \mathrm{ml}$ of distilled water at room temperature. After fiercely stirring $2 \mathrm{~h}$ and still standing $24 \mathrm{~h}$ the sol was filtered. The Si chips were ultrasonic cleaned 30 minutes by acetone and ethyl alcohol one after another then putting the chips into piranha lotion in water bath at $90{ }^{\circ} \mathrm{C}$ for an hour. The chips were finally cleaned by distilled water and pure $\mathrm{N}_{2}$.

The thin films were prepared by dip-coating method. Chips were dipped vertically into sol standing for 2 minutes and pulled vertically with a constant speed of $4 \mathrm{~mm} / \mathrm{s}$; xerogel thin films coated on chips were prepared after dried in the oven at $100^{\circ} \mathrm{C}$. Films with different thickness can be obtained by repeating the above procedure. In this work, 3 and 5 coats were applied in total for sample 1 (S1) and sample 2 (S2), respectively. Reduction of the thin films to $\mathrm{VO}_{2}$ was achieved by thermal annealing with a heating rate of $8{ }^{\circ} \mathrm{C} / \mathrm{min}$ in a vacuum furnace at $500^{\circ} \mathrm{C}$ for $2 \mathrm{~h}$ in the atmosphere of Ar.

\section{Results and discussion}

\subsection{XRD and SEM analysis}

The crystal structure and surface morphology of $\mathrm{VO}_{2}$ films were investigated by an $\mathrm{X}$-ray diffractometer using $\mathrm{Cu} \mathrm{K} \alpha$ and scanning electron microscopy.

Fig. 1 and Fig. 2 shows the X-ray diffraction patterns for $\mathrm{VO}_{2}$ films $\mathrm{S} 1$ and $\mathrm{S} 2$ on Si substrates. Two peaks at $2 \theta=27.8^{\circ}$ and $2 \theta=44.7^{\circ}$ are found both in Fig. 1 and Fig.2, corresponding to the (011) and (012) plane of $\mathrm{VO}_{2}$ phase. It is obvious that the (011) peak is much stronger than the (012) peak of $\mathrm{VO}_{2}$. The full-width-at-half-maximum (FWHM) of (011) plane peak for films S1 and S2 is $0.339^{\circ}$ and $0.351^{\circ}$, respectively. Another strong peak in both Fig. 1 and Fig. 2 at $2 \theta=69^{\circ}$ turns out to be the (400) plane of high-purity single-crystal Si substrate. According to the Scherrer's formula, the thin films have a better preferred orientation with increase of their thickness. The results of XRD analysis indicate that the thin films prepared by sol-gel are mainly consist of $\mathrm{VO}_{2}$.

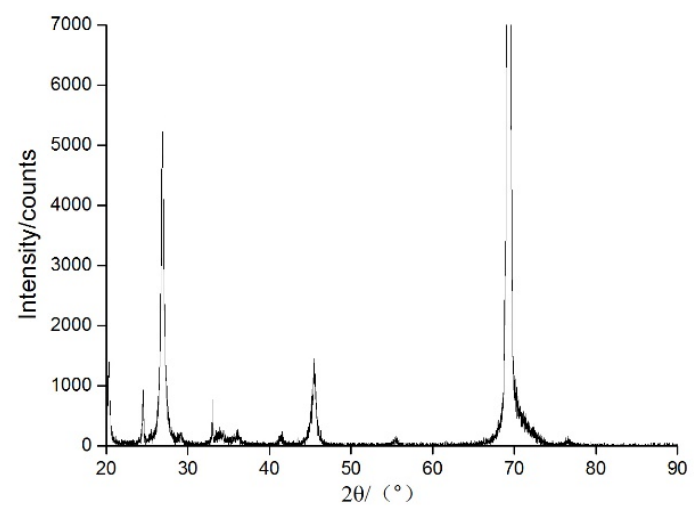

Fig.1 XRD spectra for $\mathrm{VO}_{2}$ thin films $\mathrm{S} 1$ on Si substrates 


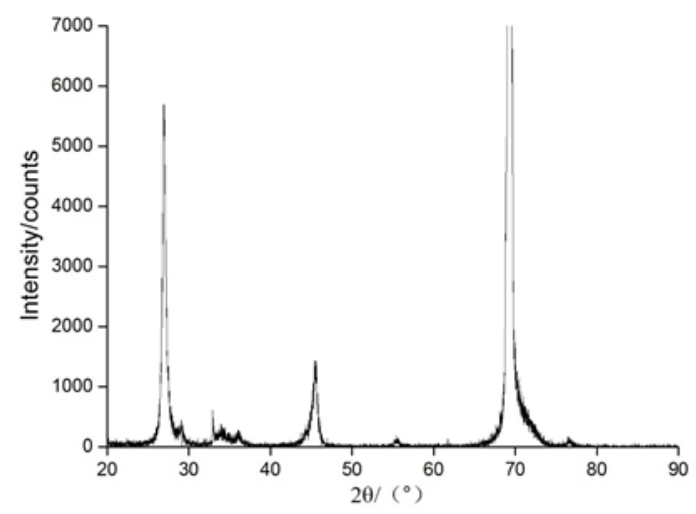

Fig.2 XRD spectra for $\mathrm{VO}_{2}$ thin films $\mathrm{S} 2$ on $\mathrm{Si}$ substrates

Fig. 3 shows the SEM results of surface morphologies for samples. There are many obvious square particles on the surface of thin films and all of them have average size. It corresponds to the monoclinic structure of $\mathrm{VO}_{2}$ at room temperature.

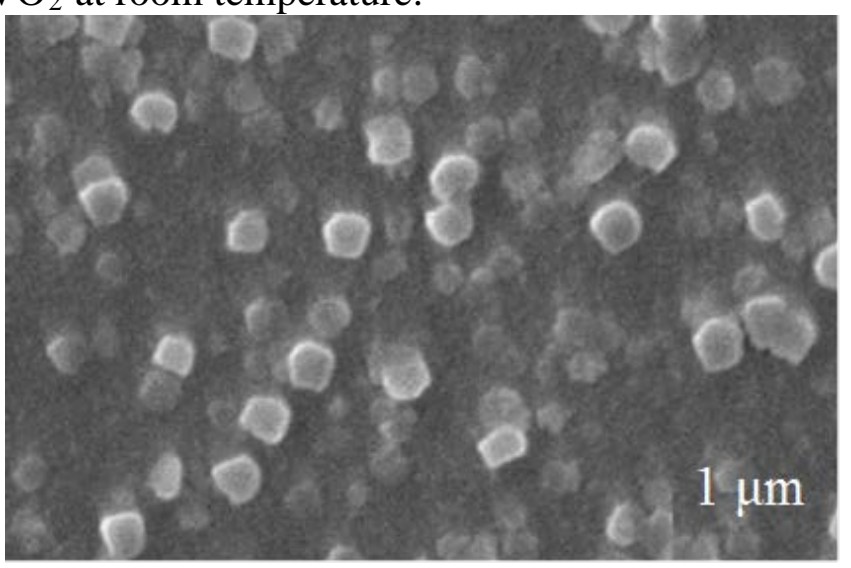

Fig.3 SEM images for VO2 thin films

\subsection{MIT properties under strong electric field}

For thin films in the research of electric field-induced phase transition with characteristics of long dynamic range, high field strength and insulation requirements, fast response speed, a phase transition testing system was manufactured based on static high voltage source and copper electrode fixture. Fig.4 exhibits the schematic diagram of the testing system.

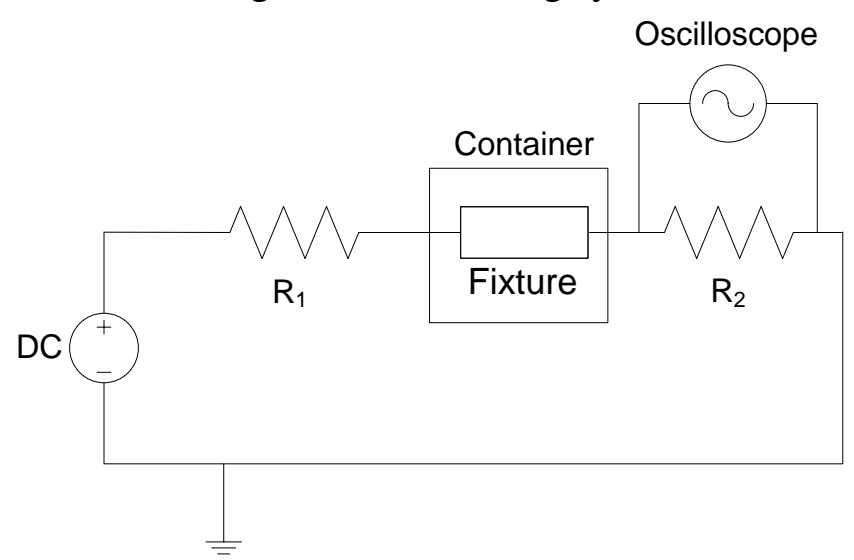

Fig. 4 Schematic diagram of test system

A static high voltage source provides the strong electric field environment with a maximum output voltage of $\pm 120 \mathrm{kV}$, security current limiting of $2.5 \mathrm{~mA}$, and step precision of $100 \mathrm{~V}$; A current limiting resistance $\mathrm{R}_{1}\left(\mathrm{R}_{1}=30 \mathrm{M} \Omega\right)$ has been used in series in order to make the current at a safe level. It's unable to measure the data of the samples under strong electric field, so a sampling resistance $R_{2}\left(R_{2}=1000 \Omega\right)$ is added to the circuit; The resistance of samples were calculated by output voltage of DC and current measured through oscilloscope in circuit. 
Fig. 5 shows the design of fixture with size of $10 \mathrm{~mm} \times 10 \mathrm{~mm} \times 50 \mathrm{~mm}$. In order to avoid point discharge [12], all other sides were made into chamfer shape except the sides which contacted to the samples. The whole device was placed in a tailor-made glass container filled with $\mathrm{SF}_{6}$ to increase its electrical insulation.

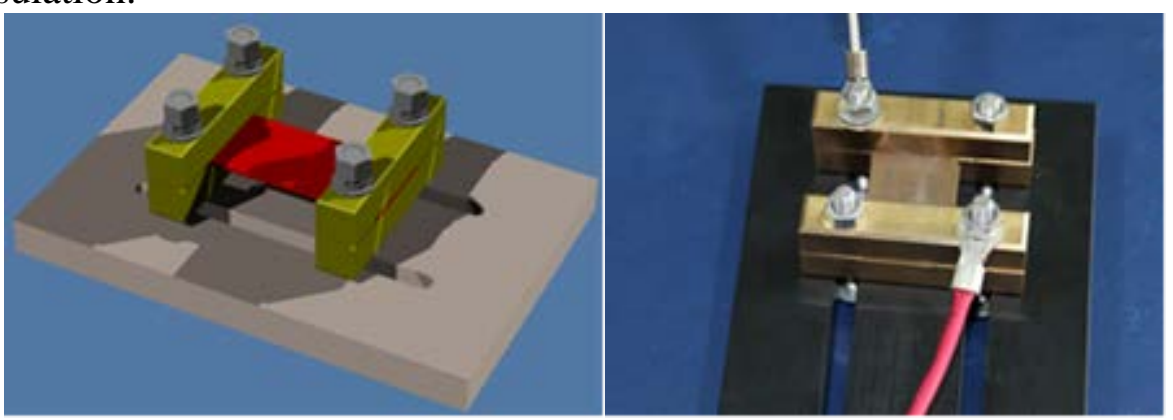

(a) fixture model

(b) real product

Fig.5 Design of copper electrode fixture

The conductivity of $\mathrm{VO}_{2}$ thin film samples under different output voltage was calculated through Eq. (1),

$$
G=\frac{1}{R}=\frac{U_{2}}{U_{0} R_{2}-U_{2} R_{1}-U_{2} R_{2}}
$$

Fig.6 exhibits the relationship between conductivity and electric field. It can be found in Fig. 6 that both S1 and S2 show an outstanding MIT property with the increase of average intensity of electric field. S1 with lower thickness shows phase transition at $500 \mathrm{kV} / \mathrm{m}$ while for S2 with larger thickness at $600 \mathrm{kV} / \mathrm{m}$. The conductivity for S1 and S2 changes more than 280 and 400 times, respectively. The variation of conductivity for S2 reaches more than 2.6 magnitudes at $700 \mathrm{kV} / \mathrm{m}$ and far from saturated. And there is still much room with the increase of external electric field.

Compared with S1, two more coats were applied for S2 leading to a larger thickness and a more complete crystal structure. That makes the $\mathrm{VO}_{2}$ thin films possess better MIT properties and nonlinear coefficient for the change of conductivity while the phase transition happens. In addition, multiple retests on both S1 and S2 show that there is no obvious variation of conductivity. The test results indicate that the $\mathrm{VO}_{2}$ thin films prepared by sol-gel method have stable and reversible field-induced MIT properties.

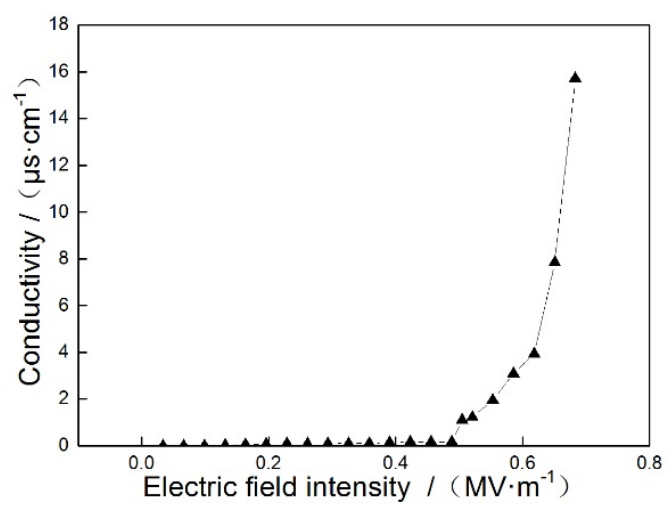

(a) S1 


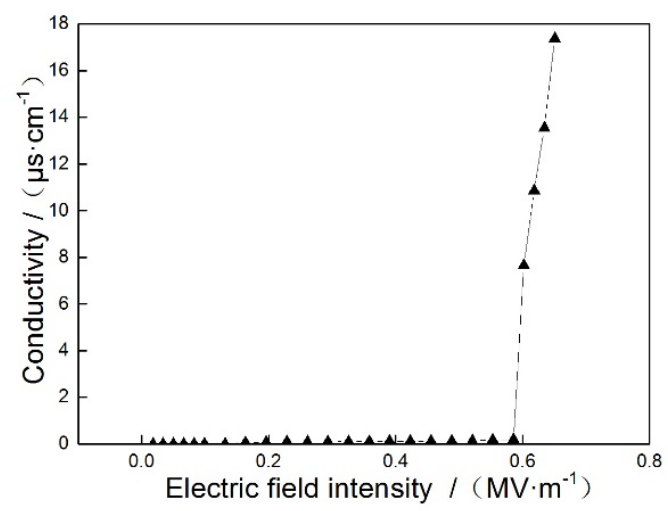

(b) S2

Fig.6 Variation tendency of conductivity of thin film samples

\section{Conclusions}

In conclusion, $\mathrm{VO}_{2}$ thin films with single-component and complete crystal structure were prepared on high-purity single-crystal Si substrates by inorganic sol-gel method. With the increase of films' thickness, change of conductivity for S2 can be more than 2.6 magnitudes and increases 50\% compared to S1.The experimental results of conductivity test indicated that $\mathrm{VO}_{2}$ thin films have the characteristics of large variation tendency, high nonlinear coefficient and stable phase transition.

\section{References}

[1] Zhang Jun,Zhong Huihuang,Yang Hanwu. Developments of pulsed power technology for driving HPM sources[J]. High Voltage Engineering, 2004, 06:45-48+53.

[2] Sun Fengju, Zeng Jiangtao, Jiang Xiaofeng, et al. Conception Design of a Long-pulsed Driver Based on FLTD and RLC Snubber[J]. High Voltage Engineering, 2009, 01:99-102.

[3] Wang Jianzhong, Xi Zhengping, Tang Huiping, et al. Research Progress of Electromagnetic Shielding Material of Metal Fiber[J]. Rare Metal Materials and Engineering, 2011, 09:1688-1692.

[4] Morin F J. Oxides which show a metal - to-insulator transition at the Neel temperature[J]. Phy. Rev. Lett., 1959, 3(1):34-36.

[5] Cui Jingzhong, Da Daoan, Jiang Wanshun. Structure characterization of vanadium oxide thin films prepared by magnetronsputtering methods[J]. Applied Structure Science, 1998, 133:225-229.

[6] Schlag H J, Scherber W. New sputter process for VO2 thin films andexamination with MIS elements and C - V - measurements[J]. Thin Solid Films, 2000, 366:28 - 31.

[7] Gang Xu, et al. Optimization of antireflection coating for VO2-based energy efficient window[J]. Solar Energy Materials \& Solar Cells, 2004, 83(1):29-37.

[8] Fang Yingcui, Chen Changqi, Zhu Wu, et al. Study on the application of VO2 in smart window[J] Vacuum, 2003(2):16-19.

[9] Lu Yuan, Ling Yongshun, Feng Yunsong, et al. Analysis of VO2 thin film intelligent protection against pulsed power infrared-laser[J]. Infrared and Laser Engineering, 2012， 11:2886-2890.

[10] Yue Fang, Huang Wanxia, Shi Qiwu, et al. Growth Mode of $\mathrm{VO}_{2}$ Films on $\mathrm{SiO}_{2}$ Substrate and Its Phase Transition Property[J]. Rare Metal Materials and Engineering, 2014, 08:1955-1958.

[11] Wang Caixiong, Tang Zhiguo, Chang Wenzhi, et al. Experimental Study on Development Characteristics of Point Discharge in GIS[J]. Power System Technology, 2011, 11:157-162. 